\title{
Estado Nutricional de Idosos Oncológicos por meio de Diferentes Métodos
}

\author{
doi: https://doi.org/10.32635/2176-9745.RBC.2018v64n2.80
}

Nutritional Assessment of Oncology Elderly by Different Methods
Evaluación Nutricional de los Ancianos Oncológicos por Diferentes Métodos

Lucivalda Pereira Magalhães Oliveira ${ }^{1}$; Taise Andrade da Anunciação²; Maria Lúcia Varjão da Costa ${ }^{3}$

Resumo

Introduçáo: $\mathrm{O}$ estado nutricional e a localizaçáo do tumor são essenciais na avaliaçáo do idoso com câncer, pois relacionam-se com morbimortalidade e impactam diretamente na sobrevida. Objetivo: Avaliar o estado nutricional segundo a mini avaliação nutricional, índice de massa corporal e circunferência da panturrilha, identificar o grau de concordância entre esses parâmetros e verificar a associação entre o estado nutricional e os diferentes tipos de câncer. Método: Estudo transversal, com pacientes idosos oncológicos internados em um hospital de referência em Salvador, Bahia. Na coleta dos dados, utilizou-se a mini avaliação nutricional (versão reduzida), questionário estruturado para coleta de dados clínicos e perfil sociodemográfico, e antropométricos. Realizaram-se análise descritiva, teste de qui-quadrado $\left(\mathrm{x}^{2}\right)$ e teste Kappa. Resultados: Observou-se que 41\%, 54,3\% e 74,3\% dos pacientes apresentavam estado nutricional adequado segundo a mini avaliação nutricional, índice de massa corporal e circunferência da panturrilha, respectivamente, com uma concordância moderada (Kappa=0,59) entre índice de massa corporal e mini avaliação nutricional. Os maiores comprometimentos do estado nutricional foram observados nos pacientes com diagnóstico de câncer de cabeça e pescoço. Conclusão: Neste estudo, a mini avaliação nutricional foi mais sensível para identificar inadequaçáo no estado nutricional quando comparada com o índice de massa corporal e circunferência da panturrilha; observou-se ainda associação entre o estado nutricional e a localização do tumor. Provavelmente, os parâmetros subjetivos da mini avaliação nutricional justificam o diagnóstico de desnutrição mais precoce, favorecendo a intervenção nutricional.

Palavras-chave: Idoso; Estado Nutricional; Avaliação Nutricional; Índice de Massa Corporal; Composição Corporal.

\begin{abstract}
Introduction: The nutritional status and localization of the tumor are essential in the evaluation of the elderly with cancer, since they are related to morbimortality and have a direct impact on survival. Objective: To evaluate the nutritional status according to the mini nutritional assessment, body mass index and calf circumference, to identify the degree of agreement between these parameters and to verify the association between the nutritional status and the different types of cancer. Method: Cross-sectional study with elderly oncology patients admitted to a referral hospital in Salvador, Bahia. Data were collected using the mini nutrition assessment (reduced version), structured questionnaire for the collection of clinical data and sociodemographic profile, and anthropometric data. Descriptive analysis, chi-square test $\left(\mathrm{x}^{2}\right)$ and Kappa test were performed. Results: It was observed that $41 \%, 54.3 \%$ and $74.3 \%$ of the patients presented adequate nutritional status according to the mini nutritional assessment, Body Mass Index and Calf Circumference, respectively, with moderate agreement (Kappa=0, 59) between Body Mass Index and mini nutritional assessment. The highest nutritional status was observed in patients diagnosed with head and neck cancer. Conclusion: In this study the mini nutritional assessment was more sensitive to identify inadequacy in the nutritional status when compared with the Body Mass Index and calf circumference; an association between nutritional status and tumor location was also observed. Probably, the subjective parameters of the mini nutritional assessment justify the diagnosis of earlier malnutrition, favoring nutritional intervention.

Key words: Aged; Nutritional Status; Nutritional Assessment; Body Mass Index; Body Composition.
\end{abstract}

Resumen

Introducción: El estado nutricional y localización del tumor son esenciales en la evaluación del anciano con cáncer, pues se relacionan con morbimortalidad e impactan directamente en la supervivencia. Objetivo: Evaluar el estado nutricional según la mini evaluación nutricional, índice de masa corporal y circunferencia de la pantorrilla, identificar el grado de concordancia entre esos parámetros y verificar la asociación entre el estado nutricional y los diferentes tipos de cáncer. Método: Estudio transversal, con pacientes ancianos oncológicos internados en un hospital de referencia en Salvador, Bahia. En la recolección de los datos se utilizó la mini evaluación nutricional (versión reducida), cuestionario estructurado para recolección de datos clínicos y perfil sociodemográfico, y antropométricos. Se realizó análisis descriptivo, prueba de chi-cuadrado $\left(\mathrm{x}^{2}\right)$ y prueba Kappa. Resultados: Se observó que 41\%, 54,3\% y $74,3 \%$ de los pacientes presentaban adecuado estado nutricional según la Mini Evaluación Nutricional, Índice de Masa Corporal y Circunferencia de la pantorrilla, respectivamente, con una concordancia moderada (Kappa=0, 59) entre índice de masa corporal y mini evaluación nutricional. Dado que los mayores compromisos del estado nutricional se observaron en los pacientes con diagnóstico de cáncer de cabeza y cuello. Conclusión: En este estudio la mini evaluación nutricional fue más sensible para identificar inadecuación en el estado nutricional cuando comparada con el Índice de Masa Corporal y Circunferencia de la pantorrilla; se observó una asociación entre el estado nutricional y la localización del tumor. Probablemente, los parámetros subjetivos de la mini evaluación nutricional justifican el diagnóstico de desnutrición más precoz, favoreciendo la intervención nutricional.

Palabras clave: Anciano; Estado Nutricional; Evaluación Nutricional; Índice de Masa Corporal; Composicion Corporal.

\footnotetext{
${ }^{1}$ Doutora pelo Programa de Medicina e Saúde da Universidade Federal da Bahia (UFBA). Professora-Adjunta do Departamento Ciência da Nutrição da Escola de Nutrição da UFBA. Salvador (BA), Brasil. E-mail: valdapm@hotmail.com. Orcid ID: https://orcid.org/0000-0003-4822-5930

${ }^{2}$ Especialista em Nutrição Clínica. Programa de Pós-Graduação da Escola de Nutrição da UFBA. Salvador (BA), Brasil. E-mail: tai.anunciacao@hotmail.com. Orcid ID: https://orcid.org/0000-0001-6207-8784

${ }^{3}$ Mestre pelo Programa de Pós-graduação em Alimentos, Nutrição e Saúde da Escola de Nutrição da UFBA. Nutricionista do HAM. Salvador (BA), Brasil. E-mail: lucia. varjao@uol.com.br. Orcid ID: https://orcid.org/0000-0002-5648-6738

Endereço para correspondência: Taise Andrade da Anunciação. UFBA. Escola de Nutrição. Rua Araújo Pinho, 32 - Canela. Salvador (BA), Brasil.
} 


\section{INTRODUÇÃO}

O acentuado envelhecimento populacional representa um grande desafio para a saúde pública, com o aumento da prevalência de doenças crônicas não transmissíveis, entre estas, o câncer ${ }^{1,2}$. Estudos brasileiros observaram que, com o aumento da idade, aumenta a porcentagem dos casos de câncer, sendo a maior ocorrência em pessoas com faixa etária entre 60 a 64 anos e 65 a 74 anos $^{3}$. $\mathrm{O}$ estado nutricional em pacientes oncológicos é fragilmente modificado e pode interferir no prognóstico do câncer, além de ter associação direta com a resposta ao tratamento ${ }^{4}$.

A desnutriçấo é comum em pacientes oncológicos, e relaciona-se com a localizaçáo, estadiamento do tumor e efeitos adversos ao tratamento antineoplásico ${ }^{5}$. O alto risco nutricional está associado, pincipalmente, à população idosa, independente do sexo, devido às alterações biológicas próprias da idade, com progressiva diminuição musculoesquelética, órgãos e líquidos corporais ${ }^{6}$. Assim, diversos parâmetros, como exame físico, laboratoriais, clínico, inquérito alimentar e dados antropométricos, devem ser utilizados para identificação do estado nutricional dessa população ${ }^{7}$. No entanto, estes devem ser sensíveis o suficiente para identificar as alteraçôes de forma precoce ${ }^{8}$.

A triagem nutricional na admissão hospitalar pode identificar precocemente o risco nutricional ou a desnutrição e melhorar o prognóstico. Entre os protocolos de triagem nutricional para pacientes oncológicos, destaca-se a Avaliação Subjetiva Global Produzida pelo Próprio Paciente (ASG-PPP), considerada específica e sensível na avaliação desses pacientes ${ }^{7,9}$. Por outro lado, existe a mini avaliação nutricional (MAN), que é uma ferramenta validada, sensível e capaz de identificar desnutrição ou risco de desnutriçáo em pacientes com idade maior ou igual a 65 anos $^{10}$.

Diante dos diferentes métodos disponíveis na identificação do estado nutricional de pacientes idosos oncológicos, e da necessidade de uma ferramenta de avaliação nutricional sensível e de aplicação simples, que possa identificar de forma precoce o risco nutricional, o presente estudo tem como objetivos avaliar o estado nutricional segundo a MAN versão reduzida, índice de massa corporal (IMC) e circunferência da panturrilha (CP); identificar o grau de concordância entre esses parâmetros; e verificar a associaçáo entre o estado nutricional e os diferentes tipos de câncer de pacientes idosos oncológicos internados em um hospital de referência em Salvador, Bahia.

\section{MÉTODO}

Trata-se de um estudo transversal realizado em um hospital oncológico de referência, na cidade de Salvador,
Bahia. O presente trabalho faz parte de um estudo multicêntrico realizado pelo Instituto Nacional de Câncer José Alencar Gomes da Silva (INCA) em 50 instituições que atendem a pacientes oncológicos em diversas cidades do Brasil e de Portugal.

A amostra consta de idosos de ambos os sexos, internados nas enfermarias de clínica médica e cirúrgica, no período de setembro e outubro de 2014. Para essa investigaçáo, foi utilizada uma amostragem não probabilística, na qual foram incluídos 371 pacientes idosos, com idade $\geq 65$ anos, portadores de tumores malignos, independente de localizaçáo ou estadiamento da doença. Foram excluídos pacientes que não tinham condiçóes de responder ao formulário da MAN, e sem acompanhante responsável capaz de responder às informações, ou aqueles que náo concordaram em assinar o Termo de Consentimento Livre e Esclarecido (TCLE). Além disso, foram excluídos pacientes que apresentavam edemas em regiáo dos membros inferiores, impossibilitando a aferição da CP.

A precisão das informaçóes colhidas foi garantida por meio de treinamento da equipe do projeto. Para coleta dos dados, utilizou-se um questionário estruturado para coleta de dados clínicos e perfil sociodemográfico, com informaçôes sobre questôes sobre data da internação, idade (anos), sexo (masculino e feminino), diagnóstico de hipertensão arterial sistêmica e diabetes mellitus, tabagismo e/ou etilismo no momento e/ou antes do internamento; motivo da internação, e dado clínico com localização do tumor. Foi considerado apenas o câncer primário para aqueles pacientes que apresentavam mais de uma localização do tumor. Para efeito de análise, a variável localização da doença foi categorizada da seguinte forma: trato gastrointestinal, mama, próstata, trato respiratório, pele, trato urinário (rim, bexiga e ureteres), cabeça e pescoço, aparelho reprodutor feminino (ovário e útero), aparelho reprodutor masculino (pênis e testículo), órgãos anexos (fígado, vesícula, pâncreas e baço) e outros (pélvis, retroperitônio e sistema ósseo).

Para avaliação do estado nutricional dos pacientes, foram utilizados o IMC, a CP e a MAN versão reduzida. Para o cálculo do IMC, foi considerado o peso e a altura do paciente, dividindo-se o peso pela altura ao quadrado. O peso corporal foi aferido com a utilização de uma balança digital calibrada (Techline ${ }^{\odot}$ ) com o paciente em pé, posicionado no centro da plataforma da balança, vestindo roupas leves e descalço. Para estimar a altura do paciente, utilizou-se uma fita inelástica, com graduação de $1 \mathrm{~mm}$, para aferir a altura do joelho, posicionando o paciente com o joelho e tornozelo em um ângulo de $90^{\circ}$, de acordo com a recomendação da World Health Organization $(\mathrm{WHO})^{10}$. Foi utilizada a estimativa da 
altura proposta por Chumlea et al. ${ }^{11}$. Para a classificação do estado nutricional dos pacientes segundo o IMC, foram usados os pontos de corte específicos para população idosa, recomendados pela Organização Pan-Americana da Saúde $(\mathrm{Opas})^{12}$, sendo considerados: baixo peso (IMC $<23 \mathrm{~kg} /$ $\left.\mathrm{m}^{2}\right)$; peso normal $\left(23<\mathrm{IMC}<28 \mathrm{~kg} / \mathrm{m}^{2}\right)$; pré-obesidade $\left(28<\mathrm{IMC}<30 \mathrm{~kg} / \mathrm{m}^{2}\right) ;$ e obesidade $\left(\mathrm{IMC}>30 \mathrm{~kg} / \mathrm{m}^{2}\right)^{12}$. Para efeito de análise, os pacientes com IMC $<23 \mathrm{~kg} / \mathrm{m}^{2}$ foram agrupados em uma única categoria "inadequado". E os pacientes com IMC $>23 \mathrm{~kg} / \mathrm{m}^{2}$ foram incluídos na categoria de "adequado".

A CP foi mensurada com o paciente sentado com os pés ligeiramente afastados e a perna em ângulo de $90^{\circ}$, posicionando a fita inelástica e flexível ao redor da panturrilha, seguindo a recomendaçáo da $\mathrm{WHO}^{11}$. Foi utilizado o ponto de corte da $\mathrm{CP} \geq 31 \mathrm{~cm}$ como adequado e $\mathrm{CP}<31 \mathrm{~cm}$ como inadequado ${ }^{12}$.

A triagem utilizando a MAN versão reduzida inclui perguntas subjetivas sobre modificação da ingestão alimentar nos últimos três meses (diminuiçáo severa, moderada ou sem diminuição), perda de peso nos últimos três meses (superior a $3 \mathrm{~kg}$, entre 1 a $3 \mathrm{~kg}$, não sabe informar, ou sem perda de peso), mobilidade (restrito ao leito ou cadeirante, deambula, mas náo sai de casa ou se locomove normal), presença de estresse psicológico ou doença aguda nos últimos três meses e problemas neuropsicológicos (demência ou depressão graves, demência leve ou sem problemas). E como informaçôes objetivas, os dados antropométricos (peso, altura e CP).

Na classificação do estado nutricional segundo a MAN, foi considerado o somatório dos escores do questionário validado por Kaiser et al. ${ }^{10}$, sendo 14 pontos o máximo a ser atingido. A estratificação do estado nutricional de acordo com a pontuação obedece à seguinte classificação: estado nutricional adequado (MAN 12-14 pontos); risco de desnutriçáo (MAN 8-11 pontos); e desnutrição (MAN 0-7 pontos). Para efeito de análise, os pacientes classificados pela MAN como risco de desnutrição e desnutrição (<12 pontos) foram agrupados em uma única categoria (Inadequado). Já os pacientes classificados como estado nutricional adequado ( $\geq 12$ pontos) foram incluídos na categoria "Adequado".

Para análise dos dados, utilizou-se o programa Statistical Package for Social Science - SPSS versão 20.0 (SPSS for Windows, 2008). O teste de qui-quadrado $\left(\mathrm{x}^{2}\right)$ foi utilizado para avaliar as associaçôes entre os estados nutricionais obtidos pela MAN vs. IMC e MAN vs. CP, bem como avaliar a associação entre estado nutricional (MAN e IMC) e a localização do tumor. O teste Kappa foi utilizado para verificar a concordância entre os diferentes métodos MAN, IMC e CP. Foi adotada a escala proposta por Landis e $\mathrm{Koch}^{14}$ com os seguintes valores de Kappa: $<0$ : grau de concordância pobre; 0 a 0,2: grau de concordância muito leve; 0,21 a 0,4 : grau de concordância leve; 0,41 a 0,6 : grau de concordância moderado; 0,61 a 0,8 : grau de concordância substancial; 0,81 a 1: grau de concordância quase perfeito. Foi adotado nível de significância de 5\% $(\mathrm{p}<0,05)$ em todas as análises.

Considerando os aspectos éticos, os voluntários foram esclarecidos dos procedimentos para a pesquisa, conforme as determinaçôes institucionais e a Resolução número 466/12 do Conselho Nacional de Saúde. A participação dos idosos se deu de forma voluntária, mediante assinatura ou impressáo digital do TCLE. Este estudo foi aprovado pelo Comitê de Ética em Pesquisa (CEP) do INCA, sob o número de parecer 688.258 , bem como pelo CEP do Hospital Aristides Maltez da Liga Baiana contra o Câncer, sob o número de parecer 925.172 .

\section{RESULTADOS}

Verifica-se, na Tabela 1, que a maioria desses pacientes era do sexo masculino (61\%), com predomínio de participantes na faixa etária entre 65 a 75 anos (74,6\%).

Ao avaliar a caracterização nutricional conforme os parâmetros utilizados, constata-se, na Tabela 1, que, de acordo com a MAN, a maioria dos pacientes apresentava estado nutricional inadequado (59,0\%). Enquanto, segundo o IMC e CP, 45,7\% e 25,7\%, respectivamente, foram classificados como inadequado, com diferenças estatisticamente significantes (MAN vs. IMC, $\mathrm{p}=0,000 \mathrm{e}$ MAN vs. CP, $\mathrm{p}=0,000)$.

$\mathrm{Na}$ Tabela 2, estão apresentadas as frequências dos itens subjetivos da MAN versão reduzida. Verifica-se que a maioria dos pacientes referia náo ter diminuído a ingestão alimentar nos últimos três meses $(65,9 \%)$, não apresentaram perda de peso $(58,1 \%)$, mobilidade normal $(80,4 \%)$ e não apresentavam problemas neuropsicológicos $(98,6 \%)$. Por outro lado, a maioria passou por um estresse psicológico ou doença aguda nos últimos três meses $(72,0 \%)$. E, ao analisar a associação entre estresse psicológico e estado nutricional segundo a MAN, verificou-se que $68,7 \%$ dos pacientes que responderam positivamente foram classificados com estado nutricional inadequado (Gráfico 1), sendo essa associaçáo estatisticamente significante $(\mathrm{p}=0,000)$.

$\mathrm{Na}$ Tabela 3, observa-se que a maioria dos pacientes com diagnóstico de câncer de cabeça e pescoço apresentava estado nutricional inadequado segundo os parâmetros avaliados, $81,2 \%$ (MAN) e 78,1\% (IMC), sendo essa associação estatisticamente significante $(\mathrm{p}=0,007 \mathrm{e}$ $\mathrm{p}=0,000$, respectivamente). Entre os pacientes com câncer gastrointestinal, 80,8\% (MAN) e 50\% (IMC) apresentavam estado nutricional inadequado, todavia a 
Tabela 1. Caracterização demográfica, clínica e estilo de vida de pacientes idosos oncológicos de um hospital de referência, Salvador - Bahia, 2014

\begin{tabular}{|c|c|c|}
\hline & Variáveis & n (\%) \\
\hline \multirow{2}{*}{ Sexo } & Masculino & $211(61,0)$ \\
\hline & Feminino & $135(39,0)$ \\
\hline \multirow{4}{*}{ Idade } & $65-75$ & $258(74,6 \%)$ \\
\hline & $76-85$ & $74(21,4 \%)$ \\
\hline & $86-95$ & $12(3,5 \%)$ \\
\hline & $96-105$ & $2(0,6 \%)$ \\
\hline \multirow{2}{*}{$\mathrm{HAS}^{1}$} & Sim & $208(60,1 \%)$ \\
\hline & Não & $138(39,9 \%)$ \\
\hline \multirow{2}{*}{$\mathrm{DM}^{2}$} & Sim & $67(19,4)$ \\
\hline & Não & $279(80,6)$ \\
\hline \multirow{2}{*}{ Etilista } & Sim & $34(9,8)$ \\
\hline & Não & $312(90,2)$ \\
\hline \multirow{2}{*}{ Tabagista } & Sim & $48(13,9)$ \\
\hline & Não & $298(86,1)$ \\
\hline \multirow[b]{2}{*}{$\mathrm{MAN}^{3}$} & Adequado ( $\geq 12$ pontos) & $142(41,0)$ \\
\hline & $\begin{array}{l}\text { Inadequado } \\
\text { (<12 pontos) }\end{array}$ & $204(59,0)$ \\
\hline \multirow{2}{*}{$\mathrm{IMC}^{4}$} & Adequado $\left(>23 \mathrm{~kg} / \mathrm{m}^{2}\right)$ & $188(54,3)$ \\
\hline & Inadequado ( $\left.\leq 23 \mathrm{~kg} / \mathrm{m}^{2}\right)$ & $158(45,7)$ \\
\hline \multirow{2}{*}{$\mathrm{CP}^{5}$} & Adequado ( $\geq 31 \mathrm{~cm}$ ) & $257(74,3)$ \\
\hline & Inadequado $(<31 \mathrm{~cm})$ & $89(25,7)$ \\
\hline \multirow{5}{*}{$\begin{array}{l}\text { Tipos de } \\
\text { câncer }\end{array}$} & Próstata & $29,2 \%$ \\
\hline & Pele & $22,8 \%$ \\
\hline & Mama & $9,5 \%$ \\
\hline & Cabeça e pescoço & $9,2 \%$ \\
\hline & Trato gastrointestinal & $7,5 \%$ \\
\hline
\end{tabular}

Legendas: ${ }^{1} \mathrm{HAS}$ : hipertensão arterial sistêmica; ${ }^{2} \mathrm{DM}$ : diabetes mellitus; ${ }^{3} \mathrm{MAN}$ : mini avaliaçáo nutricional: adequado (12-14 pontos); risco de desnutriçáo (8-11 pontos); desnutriçăo (0-7 pontos); ${ }^{4} \mathrm{IMC}:$ índice de massa corporal: adequado (IMC $>23 \mathrm{~kg} / \mathrm{m}^{2}$ ) e baixo peso $\left(\mathrm{IMC}<23 \mathrm{~kg} / \mathrm{m}^{2}\right.$ ); ${ }^{5} \mathrm{CP}$ : circunferência da panturrilha: adequado $(\mathrm{CP} \geq 31 \mathrm{~cm})$ e desnutriçáo $(\mathrm{CP}<31 \mathrm{~cm})$.

associação foi estatisticamente significante apenas segundo a MAN ( $\mathrm{p}=0,019)$. Observou-se que a MAN quando comparada com o IMC identificou um maior percentual de pacientes em risco nutricional ou desnutrição, para os cincos tipos de câncer mais prevalentes (Tabela 3).

A Tabela 4 apresenta o grau de concordância entre as classificaçóes do estado nutricional segundo o IMC e a CP, em comparação com a MAN versão reduzida. Verifica-se que o IMC apresentou um grau de concordância moderado em relação à MAN (Kappa $=0,590)$, sendo o teste estatisticamente significante $(\mathrm{p}=0,000)$. Porém, o grau de concordância entre a MAN e a CP foi considerado leve (Kappa $=0,335)$ e estatisticamente significante $(\mathrm{p}=0,000)$.
Tabela 2. Dados clínicos e nutricionais obtidos pelos cinco itens subjetivos da MAN aplicada nos pacientes idosos oncológicos de um hospital de referência, Salvador - Bahia, 2014

\begin{tabular}{|c|c|c|}
\hline & Variáveis & n (\%) \\
\hline \multirow{3}{*}{$\begin{array}{l}\text { Diminuição da } \\
\text { ingesta alimentar } \\
\text { nos últimos } 3 \text { meses }\end{array}$} & $\begin{array}{l}\text { Diminuição } \\
\text { severa }\end{array}$ & $35(10,1)$ \\
\hline & $\begin{array}{l}\text { Diminuição } \\
\text { moderada }\end{array}$ & $83(24,0)$ \\
\hline & Sem diminuição & $228(65,9)$ \\
\hline \multirow{4}{*}{$\begin{array}{l}\text { Perda ponderal nos } \\
\text { últimos } 3 \text { meses }\end{array}$} & $>3 \mathrm{~kg}$ & $51(14,7)$ \\
\hline & $\begin{array}{l}\text { Não sabe } \\
\text { informar }\end{array}$ & $45(13,0)$ \\
\hline & Entre 1 a $3 \mathrm{~kg}$ & $49(14,2)$ \\
\hline & $\begin{array}{l}\text { Sem perda de } \\
\text { peso }\end{array}$ & $201(58,1)$ \\
\hline \multirow{3}{*}{ Mobilidade } & $\begin{array}{l}\text { Restrito ao leito } \\
\text { ou cadeirante }\end{array}$ & $7(2,0)$ \\
\hline & $\begin{array}{l}\text { Deambula, mas } \\
\text { não sai de casa }\end{array}$ & $61(17,6)$ \\
\hline & Normal & $278(80,4)$ \\
\hline \multirow{2}{*}{$\begin{array}{l}\text { Estresse psicológico } \\
\text { ou doença aguda } \\
\text { nos últimos } 3 \text { meses }\end{array}$} & Sim & $249(72,0)$ \\
\hline & Não & $97(28,0)$ \\
\hline \multirow{3}{*}{$\begin{array}{l}\text { Problemas } \\
\text { neuropsicológicos }\end{array}$} & $\begin{array}{l}\text { Demência ou } \\
\text { depressão graves }\end{array}$ & $0(0)$ \\
\hline & Demência leve & $5(1,4)$ \\
\hline & Sem problemas & $341(98,6)$ \\
\hline
\end{tabular}

Legendas: ${ }^{1} \mathrm{HAS}$ : hipertensão arterial sistêmica; ${ }^{2} \mathrm{DM}$ : diabetes mellitus; ${ }^{3} \mathrm{MAN}$ : mini avaliaçấo nutricional: adequado (12-14 pontos); risco de desnutriçấo (8-11 pontos); desnutriçẫo (0-7 pontos); ${ }^{4} \mathrm{IMC}$ : índice de massa corporal: adequado (IMC $>23 \mathrm{~kg} / \mathrm{m}^{2}$ ) e baixo peso $\left(\mathrm{IMC}<23 \mathrm{~kg} / \mathrm{m}^{2}\right)$; ${ }^{5} \mathrm{CP}$ : circunferência da panturrilha: adequado $(\mathrm{CP} \geq 31 \mathrm{~cm})$ e desnutriçāo $(\mathrm{CP}<31 \mathrm{~cm})$.

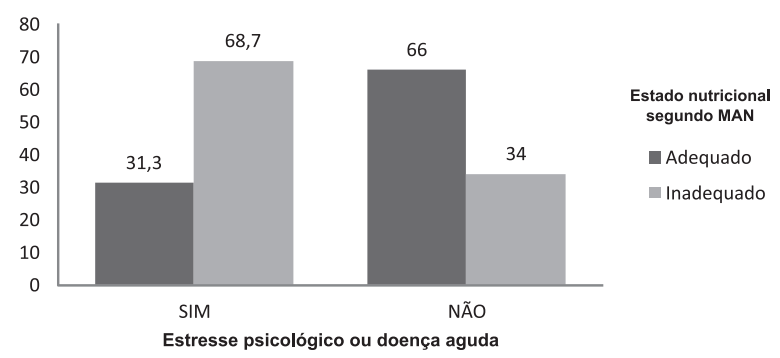

Gráfico 1. Associação entre o estado nutricional, segundo a MAN versão reduzida, e ocorrência de algum estresse psicológico ou doença aguda nos últimos três meses entre pacientes idosos oncológicos de um hospital de referência, Salvador - Bahia, 2014 *Pearson Chi-Square Test ( $\mathrm{p}$-valor $=0,000$ ) 
Tabela 3. Associação entre os cinco tipos de cânceres mais prevalentes e estado nutricional, utilizando a classificação do IMC e MAN, em pacientes idosos oncológicos de um hospital de referência, Salvador - Bahia, 2014

\begin{tabular}{|c|c|c|c|c|c|c|}
\hline \multirow{2}{*}{$\begin{array}{l}\text { Localização } \\
\text { do câncer }\end{array}$} & \multicolumn{2}{|c|}{ MAN' $1 \%)$} & \multirow[b]{2}{*}{ p-valor* } & \multicolumn{2}{|c|}{$I_{M} C^{2}(\%)$} & \multirow[b]{2}{*}{ p-valor* } \\
\hline & $\begin{array}{l}\text { Adequado } \\
\text { ( } \geq 12 \text { pontos) }\end{array}$ & $\begin{array}{l}\text { Inadequado } \\
\text { (<12 pontos) }\end{array}$ & & $\begin{array}{l}\text { Adequado } \\
\left(>23 \mathrm{~kg} / \mathrm{m}^{2}\right)\end{array}$ & $\begin{array}{l}\text { Inadequado } \\
\left(\leq 23 \mathrm{~kg} / \mathrm{m}^{2}\right)\end{array}$ & \\
\hline Câncer de próstata & 52,5 & 47,5 & 0,005 & 60,4 & 39,6 & 0,146 \\
\hline Demais & 36,3 & 63,7 & & 51,8 & 48,2 & \\
\hline Câncer de pele & 36,7 & 63,3 & 0,373 & 49,4 & 50,6 & 0,313 \\
\hline Demais & 42,3 & 57,7 & & 55,8 & 44,2 & \\
\hline Câncer de mama & 57,6 & 42,4 & 0,042 & 75,8 & 24,2 & 0,009 \\
\hline Demais & 39,3 & 60,7 & & 52,1 & 47,9 & \\
\hline Câncer de cabeça/pescoço & 18,8 & 81,2 & 0,007 & 21,9 & 78,1 & 0,000 \\
\hline Demais & 43,3 & 56,7 & & 57,6 & 42,4 & \\
\hline Câncer gastrointestinal & 19,2 & 80,8 & 0,019 & 50,0 & 50,0 & 0,644 \\
\hline Demais & 42,8 & 57,2 & & 54,7 & 45,3 & \\
\hline
\end{tabular}

Legendas: ${ }^{1} \mathrm{IMC}$ : índice de massa corporal: Adequado (IMC $>23 \mathrm{~kg} / \mathrm{m}^{2}$ ) e baixo peso (IMC $\left.<23 \mathrm{~kg} / \mathrm{m} 2\right) ;{ }^{2} \mathrm{MAN}$ : mini avaliaçăo nutricional: adequado (12-14 pontos); risco de desnutriçăo (8-11 pontos); desnutrição (0-7 pontos); *Pearson Chi-Square Test.

Tabela 4. Concordância dos diagnósticos nutricionais, segundo as classificações do IMC e CP, com a MAN, em pacientes idosos oncológicos de um hospital de referência, Salvador - Bahia, 2014

\begin{tabular}{|c|c|c|c|c|}
\hline & \multirow[b]{2}{*}{$\mathbf{N}$} & \multicolumn{2}{|c|}{ Classificação segundo a MAN³ } & \multirow{2}{*}{$\begin{array}{l}\text { Kappa* } \\
\text { (IC 95\%) }\end{array}$} \\
\hline & & $\begin{array}{c}\text { Adequado } \\
\text { ( } \geq 12 \text { pontos) }\end{array}$ & $\begin{array}{l}\text { Inadequado } \\
\text { (<12 pontos) }\end{array}$ & \\
\hline \multicolumn{5}{|c|}{ Classificação segundo a IMC ${ }^{1}$} \\
\hline Adequado $\left(>23 \mathrm{~kg} / \mathrm{m}^{2}\right)$ & 188 & $129(68,6 \%)$ & $59(31,4 \%)$ & 0,590 \\
\hline Inadequado $\left(\leq 23 \mathrm{~kg} / \mathrm{m}^{2}\right)$ & 158 & $13(8,2 \%)$ & $145(81,8 \%)$ & $P=0,000$ \\
\hline \multicolumn{5}{|c|}{ Classificação segundo a CP2 } \\
\hline Adequado $(\geq 31 \mathrm{~cm})$ & 257 & $137(53,3 \%)$ & $120(46,7 \%)$ & 0,335 \\
\hline Inadequado (<31 cm) & 89 & $5(5,6 \%)$ & $84(94,4 \%)$ & $P=0,000$ \\
\hline Total & 346 & 142 & 204 & \\
\hline
\end{tabular}

Legendas: ${ }^{1} \mathrm{IMC}$ : índice de massa corporal: adequado (IMC $\left.>23 \mathrm{~kg} / \mathrm{m} 2\right)$ e baixo peso (IMC $\left.<23 \mathrm{~kg} / \mathrm{m}^{2}\right)$; ${ }^{2} \mathrm{CP}$ : circunferência da panturrilha: adequado $(\mathrm{CP} \geq 31 \mathrm{~cm}) \mathrm{e}$ desnutriçâo $(\mathrm{CP}<31 \mathrm{~cm}) ;{ }^{3} \mathrm{MAN}$ : mini avaliação nutricional: adequado (12-14 pontos); risco de desnutrição (8-11 pontos); desnutriçăo (0-7 pontos).

*Escala proposta por Landis e Koch (1977), Kappa: < 0: grau de concordância pobre; 0 a 0,2: grau de concordância muito leve; 0,21 a 0,4: grau de concordância leve; 0,41 a 0,6: grau de concordância moderado; 0,61 a 0,8: grau de concordância substancial; 0,81 a 1: grau de concordância quase perfeito ${ }^{14}$.

\section{DISCUSSÃO}

No presente estudo, observou-se predominância do sexo masculino, sendo a faixa etária mais prevalente 65 a 75 anos, o que pode justificar o câncer de próstata ser o mais prevalente neste estudo. Resultados semelhantes foram encontrados por Sousa-Muńoz et al. que avaliaram a frequência de hospitalizações de pacientes idosos com diagnóstico de câncer ${ }^{15}$.

Nota-se que houve uma baixa prevalência de etilismo e tabagismo na população deste estudo. Todavia, essa informação pode estar subestimada, uma vez que o questionamento foi em relaçấo ao momento atual, não sendo considerado o histórico desses hábitos. Vale ressaltar que o câncer de cabeça e pescoço foi o terceiro mais frequente nessa população, e sabe-se que o tabaco é o principal fator de risco para o desenvolvimento do câncer de cabeça e pescoço, podendo ser intensificado quando associado ao etilismo ${ }^{16}$.

Em relaçáo ao estado nutricional, a MAN, quando comparada com o IMC e CP, identificou um maior número de pacientes com estado nutricional inadequado (risco nutricional e desnutrição). Resultados semelhantes foram encontrados em outros estudos, os quais observaram um maior percentual de risco nutricional e desnutrição obtido pela MAN quando comparado com o IMC ${ }^{17,18}$.

Acredita-se que a presença de dados subjetivos presentes no protocolo da MAN favorece o diagnóstico precoce de risco nutricional. Neste estudo, a variável subjetiva da MAN "passou por um estresse psicológico ou uma doença aguda nos últimos três meses", apresentou alto percentual e associação estatisticamente significativa com o estado nutricional. Achado este que pode ser justificado pelo fato de o paciente oncológico, principalmente idoso, 
apresentar desordens biopsicossociais e depressão que estão intimamente relacionadas com o processo dessa doença e, consequentemente, com o declínio nutricional e da qualidade de vida ${ }^{19,20}$.

Pode-se observar que resultados semelhantes também foram obtidos pelo estudo multicêntrico desenvolvido pelo INCA, no qual esta pesquisa está inserida. No estudo maior, foram inseridos 3.257 pacientes (entre as instituições do Brasil e Portugal); destes, $73 \%$ apresentavam desnutrição e risco nutricional segundo a MAN, enquanto $60,6 \%$ e $64,6 \%$ apresentavam estado nutricional adequado segundo IMC e CP, respectivamente ${ }^{21}$.

No presente estudo, verificou-se associação entre o estado nutricional e a localizaçáo do tumor, por meio da MAN e IMC. Os resultados indicam que, para ambos os parâmetros (MAN e IMC), a maioria dos pacientes com diagnóstico de câncer de cabeça e pescoço apresentava risco nutricional ou desnutrição quando comparados com os demais tipos de câncer, seguidos pelo câncer gastrintestinal. Marques et al..$^{22} \mathrm{e}$ Thieme et al. ${ }^{23}$ observaram que, entre os tipos de câncer, os localizados no pâncreas, estômago e cabeça/pescoço são os que apresentam uma maior prevalência de desnutrição (30\% a $80 \%)$. Ressalta-se que a localização do tumor acarreta em sintomas clínicos e efeitos adversos diferentes, refletindo diretamente no estado nutricional ${ }^{8}$.

Ao avaliar o grau de concordância entre os métodos de avaliaçáo segundo a MAN e IMC, verificou-se um grau de concordância moderado entre esses parâmetros; porém, entre a MAN e CP, o grau de concordância é leve. Resultados semelhantes foram encontrados por Leandro-Merhi et al. ${ }^{24}$ que, ao avaliarem o nível de concordância do diagnóstico nutricional de pacientes idosos hospitalizados (idade $\geq 60$ anos), utilizando MAN vs. IMC e MAN vs. CP, identificaram que o IMC apresentou um grau de concordância moderada com o método da MAN (kappa $=0,44)$ e a CP apresentou um grau de concordância leve com a MAN (kappa=0,29). Vale ressaltar que os autores avaliaram idosos hospitalizados não oncológicos.

Ferreira et al. ${ }^{25}$, ao compararem diferentes métodos de avaliação do estado nutricional (IMC, MAN e ASG) em idosos hospitalizados, concluíram que existem algumas limitaçôes ao diagnosticar o estado nutricional pelo IMC. Isso porque esse método antropométrico não leva em consideração as questôes fundamentais quando se trata da avaliação nutricional geriátrica, como capacidade funcional, depressão e ingestão alimentar. Além disso, vale ressaltar que a utilização de indicadores antropométricos para determinar o estado nutricional de idosos oncológicos, apesar de amplamente utilizados, apresentam limitaçóes, uma vez que o próprio processo de envelhecimento altera a composição corporal e as complicações clínicas podem dificultar a mensuração desses indicadores. Questóes estas que são contempladas em métodos subjetivos de triagem nutricional como a MAN.

$\mathrm{O}$ fato de a MAN pontuar com diferentes pesos esses parâmetros subjetivos pode influenciar fortemente no resultado alcançado por esse método, independente do estado nutricional obtido pela antropometria ${ }^{24}$. Os parâmetros subjetivos da MAN são fundamentais, pois podem explicar o motivo da desnutrição (reduzida ingestão alimentar, perda de peso, estresse psicológico ou dificuldade de mobilização), favorecendo o diagnóstico precoce e uma intervenção nutricional especializada.

Como limitações do estudo, tem-se a inclusão de idosos com idade acima de 65 anos, que foi critério do estudo multicêntrico. Além disso, não foi possível a utilização de outros parâmetros antropométricos como dobras cutâneas, circunferência do braço, bem como outros métodos de triagem nutricional como ASG-PPP.

\section{CONCLUSÃO}

Neste estudo, a MAN, quando comparada com o IMC e a CP, identificou maior número de pacientes com estado nutricional inadequado; observou-se associação entre localização dos tumores e estado nutricional, tanto pela MAN quanto pelo IMC. Com grau de concordância moderado entre a MAN e o IMC.

Acredita-se que os parâmetros subjetivos presentes na MAN auxiliam na identificação do diagnóstico nutricional de forma precoce, favorecendo a intervenção nutricional. É essencial uma avaliação nutricional detalhada e completa, com a utilização de instrumentos adequados para a população avaliada, a fim de detectar precocemente o paciente em risco nutricional ou desnutrido.

\section{CONTRIBUIÇÕES}

Lucivalda Pereira Magalhães Oliveira trabalhou na análise dos dados e na elaboração do artigo. Taise Andrade da Anunciação trabalhou na elaboração do artigo. Maria Lúcia Varjão da Costa trabalhou na avaliação dos pacientes, coleta de dados e conduçáo da pesquisa.

\section{DECLARAÇÃO DE CONFLITO DE INTERESSES}

Nada a Declarar.

\section{REFERÊNCIAS}

1. Marengoni A, Angleman S, Melis R, Mangialasche F, Karp A, Garmen A, Meinow B, Fratiglioni L. Aging with multimorbidity: a systematic review of the literature. Ageing Res Rev. 2011; 10(4):430-9. 
2. Burns ER, Stevens JA, Lee R. The direct costs of fatal and non-fatal falls among older adults - United States. J Safety Res. 2016; 58:99-103.

3. Instituto Brasileiro de Geografia e Estatística. Pesquisa Nacional de Saúde 2013. Rio de Janeiro: IBGE; 2014.

4. Gomes NS; Maio R. Avaliação Subjetiva Global Produzida pelo Próprio Paciente e Indicadores de Risco Nutricional no Paciente Oncológico em Quimioterapia. RBC. 2015; 61(3): 235-242.

5. Poltronieri, Taiara Scopel; Tusset, Cíntia. Impacto do tratamento do câncer sobre o estado nutricional de pacientes oncológicos: atualização da literatura. Rev. bras. ciênc. saúde. 2016; 20(4):327-332.

6. Santos PAS, Cunha TRS, Cabral EK, Soares BLM, Maio R, Burgos MGPA. Triagem Nutricional por meio do MUST no Paciente Oncológico em Radioterapia. RBC. 2016; 62(1):27-34.

7. Biangulo BF, Fortes RC. Métodos subjetivos e objetivos de avaliação do estado nutricional de pacientes oncológicos. Com. Ciências Saúde. 2013; 24(2):131-44.

8. Poziomyck AK, Fruchtenicht AVG, Kabke GB, Volkweis BS, Antoniazzi JL, Moreira LF. Reliability of nutritional assessment in patients with gastrointestinal tumors. Rev. Col. Bras. Cir. 2016; 43 (3):189-197.

9. Gonzalez MC, Borges LR, Silveira DH, Assunção MCF, Orlandi SP. Validação da versão em português da avaliação subjetiva global produzida pelo paciente. Rev Bras Nutr Clin 2010; 25(2):102-8.

10. Kaiser MJ, Bauer JM, Ramsch C, Uter W, Guigoz Y, Cederholm T, et al. Validation of the Mini Nutritional Assessment Short-Form (MNA ${ }^{\oplus}$-Sf): A Practical Tool For Identification of Nutritional Status. The J Nutr Health Aging. 2009; 13 (9):782-8.

11. World Health Organization. Technical Report Series, 854. In: Physical status: the use and interpretation of anthropometry. Genebra: WHO; 1995. p. 375-409.

12. Chumlea WC, Roche AF, Steinbaugh ML. Estimating stature from knee height for persons 60 to 90 years of age. J Am Geriatr Soc 1985; 33: 116-20.

13. World Health Organization. Encuesta multicentrica: salud, bien estar y envejecimiento (SABE) en América Latina y el CaribIn: Anales da 36ª Reunión del Comité Asesor de Investigaciones em salud. e. Washington (DC): WHO; 2001.

14. Landis JR; Koch GG. The measurement of observer agreement for categorical data. Biometrics. 1977; 33(1):159-74.

15. Sousa-Muñoz RL, Formiga MYQ, Silva AEVF, Silva MBL, Vieira RC, Galdino MM, et al. Hospitalizações por neoplasias em idosos no âmbito do sistema único de saúde na Paraíba/Brasil. Revista Saúde e Pesquisa 2015; 8(3): 479-91.

16. Chavoni RC, Silva PB, Ramos GHA. Diagnóstico nutricional de pacientes do serviço de cabeça e pescoço e sua relação com a disfagia em um hospital oncológico do Paraná. Rev. Bras. Cir. Cabeça Pescoço. 2014; 43(1):35-41.

17. Deon RG, Goldim JR. Comparação entre Diferentes Métodos de Avaliação Nutricional em Idosos Institucionalizados e não Institucionalizados. Perspectiva, Erechim. 2016; 40(149):63-71.

18. Morais FT, Campos IC, Lessa NMV. Diagnóstico Nutricional em idosos hospitalizados. Nutrir Gerais. 2010; 4(7):637-651.

19. Lera AT, Miranda MC, Trevizan LLB, Antonangelo DV, Zanellato, RM, Tateyama, LTC, Fede ABS, et al. Aplicação do instrumento termômetro de estresse em pacientes idosos com câncer: estudo piloto. Rev Bras Clin 2011; 9(2):112-6.

20. Santos CA, Ribeiro AQ, Rosa COB, Ribeiro RCL. Depressão, déficit cognitivo e fatores associados à desnutrição em idosos com câncer. Ciênc Saúde Colet. 2015; 20(3):751-60.

21. Instituto Nacional de Câncer José de Alencar Gomes da Silva. Inquérito luso-brasileiro de nutrição oncológica do idoso: um estudo multicêntrico. Rio de Janeiro: INCA; 2015.

22. Marques DC, Stringuini MLF, Fornes NS. Suplementação de ácidos graxos ômega-3, estado nutricional e qualidade de vida de pacientes com câncer gastrintestinal: estudo duplo-cego, randomizado e placebo controlado. Rev Med Minas Gerais, 2013; 23(1):39-46.

23. Thieme RD, Cutchma G, Chieferdecker MEM, Campo ACL. O índice de risco nutricional (nutritional risk índex) é preditor de complicação pós-operatória em operaçóes do aparelho digestivo ou parede abdominal. Arq Bras Cir Dig, 2013; 26(4): 286-292.

24. Leandro-Merhi VA Aquino JLB, Camargo JGT. Agreement between body mass index, calf circumference, arm circumference, habitual energy intake and the MNA in hospitalized elderly. The J Nutri, Health Aging. 2012; 16 (2):128-32.

25. Ferreira ACD, Barichello GB, Silva VL, El-Kik RM. Comparação entre métodos de avaliação do estado nutricional em idosos hospitalizados. PUCRS Journals. 2010; 3(1):1-17. 